\title{
NOTATION, SYMBOLS
}

1. $(\Omega, F, \mu)$ a measure space (most of the time isomorphic to the unit interval) $\Omega=\{\omega\}$

2. $A, B, C, D, E, F, M, N, \quad$ measurable sets

3. $P, Q$, $\mathrm{R}, \mathrm{S}$ partitions of $\Omega$ into finite or countable

4. G, $G_{1} \ldots$ sub-o-fields of $\mathrm{F}$.

5. $\mu(A / G)(\omega)$ the conditional probability of A given $G$

6. $E(f / G)(\omega)$ the conditional expectation of $f(\omega)$ given $G$

7. $\quad h(P)=-\sum_{k} \mu\left(P^{i}\right) \log \mu\left(P^{i}\right)$, where $P=\left(P^{l} \ldots P^{k} \ldots\right)$

8. $\quad P / A=\left(P^{I} \cap A, \ldots P^{k} \cap A \ldots\right)$

9. $h(P / A)=\sum_{k} \mu\left(P^{k} / A\right) \log \mu\left(P^{k} / A\right)$

10. $\quad h(P / Q)=\sum_{j} \mu\left(Q^{j}\right) h\left(P / Q^{j}\right)$

11. $\quad I(P)(\omega)=-\log \mu\left(P^{k}\right), \omega \in P^{k}$

12. $I(P / Q)(\omega)=-\log \mu\left(P^{k} / Q^{j}\right), \omega \in P^{k} \cap Q^{j}$.

13. $T, \hat{T}, R \ldots$ iransformations

14. $\quad(P)_{m}^{n}=\prod_{i=\mathbb{m}}^{n} T^{i}$.

15. $\quad F(P)=(P)_{-\infty}^{\infty}$ if $_{\text {case }} T$ is invertible, or $(P)_{-\infty}^{0}$ in the non-invertible

16. $\quad \operatorname{Tail}(P)=\bigcap_{n=0}^{\infty}(P)_{-\infty}^{-n}$

17. $P \perp Q, \quad P$ is independent of $Q$

18. $P \stackrel{Q}{\perp} Q$ : Pis $\varepsilon$ - independent of $Q$.

19. A transfirmation $T$ is automorphism if it is measure presering and invertible.

20. A set $A$ is trivial if $\mu(A)=0$ or $\mu\left(A^{c}\right)=0$

$P$ or $G$ are trivial if all their sets are trivial. 